\title{
Diversidad, composición y estructura del bosque certificado de la concesión MADERACRE S.A.C, Madre de Dios, Perú
}

\section{Diversity, composition, and structure the forest certificated of the concession MADERACRE S.A.C, Madre de Dios, Peru}

Benjamín R. Chambi Pacompía

Facultad de Ciencias Agrarias de la Universidad Nacional de San Antonio Abad del Cusco (Sede Madre de Dios). Jirón San Martin Nro.451,ProvinciaTambopata,Región Madre deDios,Perú.bchambip@gmail.com

\section{Resumen}

La presente investigación se realizó en el bosque de la concesión MADERACRE S.A.C, donde se estableció cuatro parcelas de 1 hectáreas cada una, en estas se evaluaron los árboles con DAP $\geq 10 \mathrm{~cm}$. Se calculó la diversidad, estructura, composición y similaridad de especies. Se registraron 2020 individuos en total, con 110 a 146 especies por parcela, totalizando a 277 especies, 94 géneros y 47 familias. Las familias con alto número de especies son Fabaceae, Malvaceae, Annonaceae, Moraceae, Rubiaceae, Lauraceae, Meliaceae, Sapotaceae y Salicaceae, entre los géneros: Inga, Trichilia, Pouteria, Ocotea, Acacia, Casearia, Cordia, Neea y Zanthoxylum. Las especies con mayor abundancia son; Drypetes amazonica, Attalea phalerata, Quararibea wittii, Trichilia sp1 y Pseudolmedia laevis. por ha. En promedio se registró 505 tallos/ha, con un área basal que oscila entre $21.91 \mathrm{~m}^{2} /$ ha a $28.3 \mathrm{~m}^{2} /$ ha resultado un promedio de $25.11 \mathrm{~m}^{2} /$ ha. Las especies con mayor IVI fueron: Attalea phlerata, Drypetes amazonica, Trichilia pallida, Pseudolmedia laevis, Ceiba sp1, Trichilia sp1, Quararibea wittii, Brosimum alicastrum, Pseudobombax septenatum, Celtis schippii, Spondias mombin, Cavanillesia umbellata, Drypetes sp1 y Rinorea lindeniana. La estructura vertical del bosque exhibe la forma de "J" invertida y horizontalmente toma la forma de una campana. Finalmente, la composición florística entre los sitios evaluados muestra baja similaridad de especies forestales. Los resultados obtenidos permitieron a MADERACRE S.A.C, incorporar a su Plan de Manejo Forestal.

Palabras clave: bosque certificado, manejo forestal, caracterización florística, Perú.

\section{Abstract}

The present investigation was carried out in the forest of the MADERACRE S.A.C concession, where four hectares of forest were evaluated. The diversity, structure, composition and similarity of forest species were calculated using the mathematical indexes. In total, there were 2020 individuals distributed in 277 species, 94 genera and 47 families. The families with a high number of species are Fabaceae, Malvaceae, Annonaceae, Moraceae, Rubiaceae, Lauraceae, Meliaceae, Sapotaceae and Salicaceae. And among the genera: Inga, Trichilia, Pouteria, Ocotea, Acacia, Casearia, Cordia, Neea and Zanthoxylum. The species with high abundance are; Drypetes amazonica, Attalea phalerata, Quararibea wittii, Trichilia sp1 and Pseudolmedia laevis. It has registered from 110 to 146 species per ha. On average, 505 stems / ha were recorded, with a basal area ranging from $21.91 \mathrm{~m} 2$ / ha to $28.3 \mathrm{~m} 2$ / ha, resulting in an average of $25.11 \mathrm{~m} 2 /$ ha. The species with high ecological weight are: Attalea phalerata, Drypetesn amazonica, Trichilia pallida, Pseudolmedia laevis, Ceiba sp1, Trichilia sp1, Quararibea wittii, Brosimun alicastrum, Pseudobombax septenatum, Celtis schippii, Spondias mombin, Cavanillesia umbellata, Drypetes sp1 and Rinorea lindeniana. The vertical structure of the forest exhibits the shape of "J" inverted and horizontally takes the form of a bell. Finally, the floristic composition among the among the sites evaluated shows low similarity of forest species. This results permited developing to MADERACRE S.A.C, incorpor management planes.

Kew words: certified forest, forest management, floristic characterization, Peru. 


\section{Introducción}

La región de Madre de Dios cuenta con 26 formaciones vegetales (Encarnación et al. 2008), y estas últimas décadas fueron estudiados a través de inventarios florísticos (Pitman et al. 1999, Pitman et al. 2001, Pitman et al. 2002, Rodríguez et al. 2003, Silman et al. 2005). Asimismo, de acuerdo a su zonificación forestal cuenta con 2'522,141.00 ha, destinadas para el aprovechamiento forestal, las cuales están distribuidas según el criterio fisiográfico en 13 tipos de bosque de producción permanente (INRENA 2003).

En el año 2001, la empresa maderera MADERACRE S.A.C, bajo la modalidad de concesión forestal maderable logra obtener 49,209.00 ha., distribuidas en bosques de Colina Baja fuerte (INRENA 2003) y bosque con paca (Guadua spp) (Encarnación et al. 2008). Posteriormente en el año 2005, voluntariamente logró la certificación internacional del FSC (Forest Stewardship Council) y del CCB (Climate, Comunity and Biodiversity), que consolida su compromiso con el Manejo Forestal responsable. MADERACRE S.A.C viene implementando los Estándares de Certificación del Manejo Forestal para Productos Maderables en Bosques de la Amazonía Peruana (Consejo Peruano de Certificación Forestal Voluntaria 2002). Y realiza actividades de monitoreo y evaluación del bosque según: la intensidad y escala de manejo forestal, la condición del bosque, el rendimiento de los productos forestales, la cadena de custodia, actividad de manejo y, sus impactos sociales y ambientales. Asimismo, tiene el interés en conocer la tasa de crecimiento de las especies maderables, el stock de la regeneración natural, dinámica del bosque (Toledo et al. 2001; Poorter et al.-
-2001, Manzanero \& Pinelo 2004), monitoreo de fauna silvestre, Bosque de Alto Valor de Conservación (BAVC) (WWF 2004), entre otras investigaciones que ayudan a complementar insumos para el monitoreo ecológico del bosque certificado.

Para cumplir con este propósito en la región amazónica se han establecido Parcelas Permanentes de Monitoreo en bosques primarios (Pitman et al. 1999, Ter Steege et al. 2000, Pitman, et al. 2001, Toledo et al. 2001, Pitman et al. 2002, Díaz 2007, Cano \& Stevenson 2009), los cuales contribuirán más de 1,640 ha., para conocer los patrones y monitorear el reemplazo de árboles (Phillips et al. 2004), funciones (Ter Steege et al. 2006) y dinámica de ecosistemas (Vallejos-Joyas et al. 2005), asimismo genera información con respecto a las tasas de crecimiento dasométricos (Manzanero \& Pinelo 2004) y permite modelar la dinámica volumétrica de madera a largo plazo, y es un insumo trascendental para la actualización del PGMF de la citada empresa.

La presente investigación tuvo como objetivos: a) determinar la diversidad de especies de flora en las parcelas permanentes de muestreo, b) conocer la composición de especies en la comunidad arbórea y c) determinar la estructura de las especies y familias de flora.

\section{Materiales y Métodos}

\section{Área de estudio}

El bosque de la Concesión MADERACRE S.A.C se ubica en la margen derecha del rio ACRE y en lado SUR OESTE a $28 \mathrm{~km}$ de la capital del distrito de Iñapari, provincia Tahuamanu, región Madre de Dios, Perú. Dicho distrito, es frontera con Brasil y Bolivia. 
El área está a $250 \pm 30$ m.s.n.m. y la fisiografía está conformada por colinas bajas (INRENA 2003) moderadamente a fuertemente disectadas (Encarnación et al. 2008) con suelos superficiales de textura franca y arcilla en el subsuelo, susceptibles a la erosión en sitios abiertos y geológicamente se ubica en la formación Madre de Dios y Maldonado (GOREMAD \& IIAP 2009). Según Britto (2017) corresponde a bosques no estacionales e inundables denominada "selva baja" o bosques húmedos Amazónicos (BHA). Donde se registra temperatura promedio de $24.6^{\circ} \mathrm{C}$ con variación hasta $7^{\circ} \mathrm{C}$ y $68.3 \%$ de humedad relativa (INADE 2007).

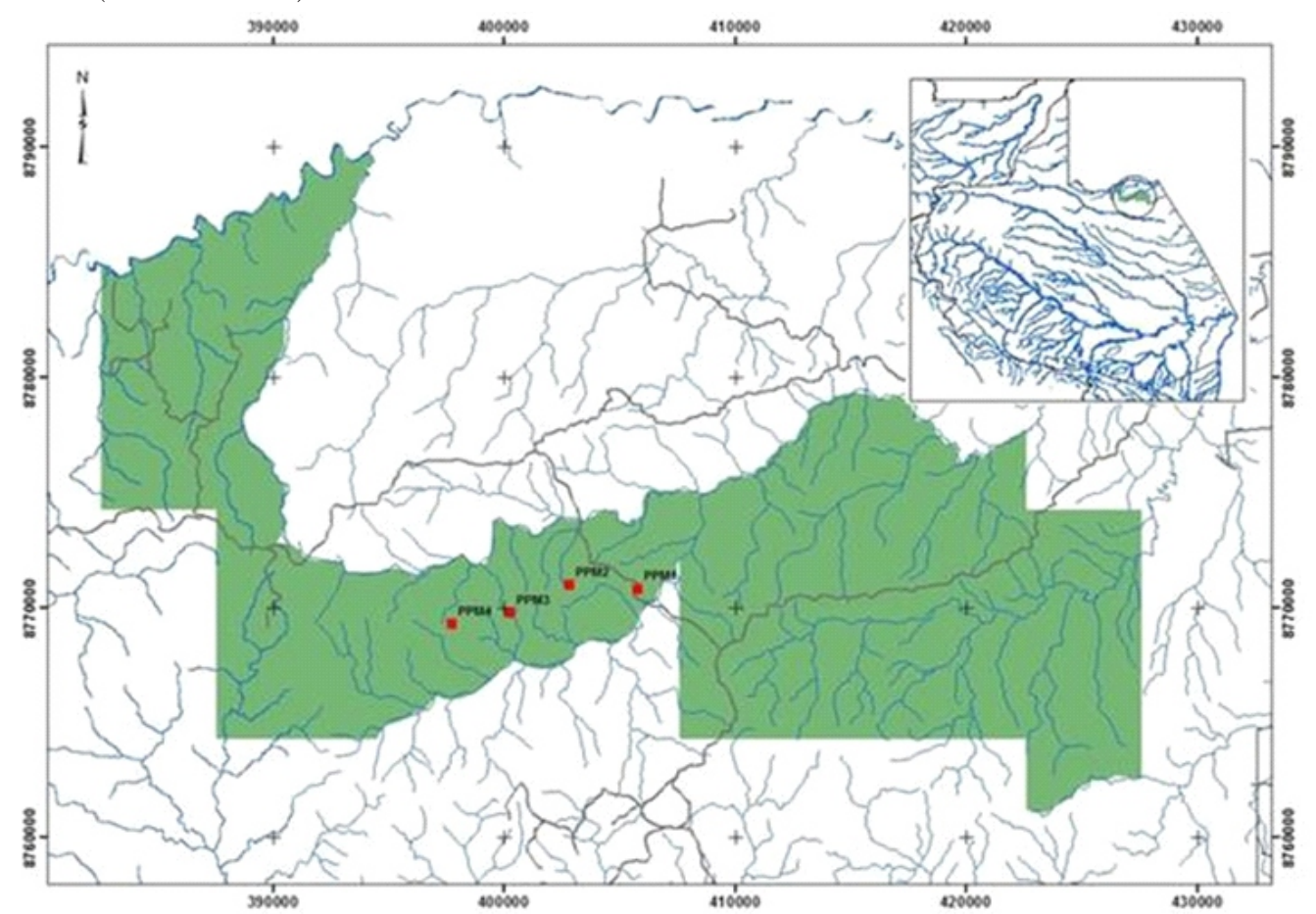

Figura 1. Ubicación de las parcelas en la concesión forestal MADERACRE S.A.C., Madre de Dios, Perú.

\section{Instalación de parcelas y evaluación de la flora}

Cada PPM fue dividida en 25 subparcelas de $20 \mathrm{~m} \times 20 \mathrm{~m}\left(400 \mathrm{~m}^{2}\right)$, donde se registró todos
La precipitación promedio anual es de $1647.1 \mathrm{~mm}$. (INADE 2007) y puede variar desde el mes de abril hasta agosto (IIAP 2001).

La instalación de Parcelas Permanentes de Monitoreo (PPM) fueron ubicadas dentro de las Parcelas de Corta Anual con aprovechamiento maderable (PCA) Nro. 8 y PCA Nro. 9, localizadas en las coordenadas UTM (WGS 84, 19S); PPM1: 405795 (E), $8770803(\mathrm{~N})$, PPM2: 402823 (E), 8770973 (N). PPM3: 400280 (E), 8769780 (N), PPM4: 397765 (E), $8769279(\mathrm{~N})$. Figura 1. 
Phillips et al. 2002). Con el propósito de conocer la altura de los árboles, se estimó la altura en base a una vara de $5 \mathrm{~m}$ considerando la complejidad del bosque tropical (Malleux 1975). La herborización de las colectas de flora fue tratada según metodología tradicional de sistemática y taxonomía vegetal (Lawrence 1951, Lot \& Chiang 1986). Asimismo, fueron comparadas con muestras botánicas digitalizadas de Herbarium Field Museum (http://fm1.fieldmuseum.org/vrrc/) y Botanical Research Institute of Texas-BRIT) (http:/ / atrium.andesamazon.org/digital_her barium.php). Todos los nombres científicos fueron revisados y actualizados considerados en el IPNI (International Plant Name Index: http: / /www.ipni.org/) y lista de especies de flora del Perú (Bracko \& Zaruchi, 1993).

\section{Análisis de datos}

\section{Diversidad}

Calcule los índices de Shannon (H) y Alfa Fisher (AF) (Moreno 2001; Mateucci \& Colma 1982), el cual fue calculado en el software Past v 1.41 (Hammer et al. 2001).

Para robustecer nuestras estimaciones sobre la riqueza de especies de árboles para el área de estudio utilizamos los indicadores MAO TAO, ACE, ICE, CHAO1, CHAO2, JACK1 y JACK2, los cuales fueron calculados con el software Stimates v7.5.0.

Estructura del bosque: Se aplicó formulas usadas por Campbell et al (1986) y Pitman et al (2001): para conocer la dominancia se usó el área basal de cada árbol $\left(\left(\underline{\pi \text { DAP }^{2}}\right) / 4\right)$. El Índice de Valor de Valor de importancia (IVI) de especies se calculó en base: Frecuencia relativa ( $\mathrm{N}^{\mathrm{ro} .}$ Árboles de A / $\mathrm{N}^{\mathrm{o}}$ de parcela), Densidad relativa de la especie o familiaA, se calcula en base al $\mathrm{N}^{\mathrm{ro}}$ Árboles de-
$\mathrm{A} / \mathrm{N}^{\circ}$ árboles en parcela) x 100, Dominancia relativa de la especie o familia $\mathrm{A}$ ((Área basal de A / Área basal total de árboles en la parcela) x 100).

Organización estructural: La organización estructural vertical se realizó en base a un histograma, donde fue agrupada en intervalos de $5 \mathrm{~m}$. de altura, de similar manera, se realizó para organización horizontal, el cual fue agrupado en intervalos de $10 \mathrm{~cm}$ de DAP.

\section{Análisis Multivariado}

Agrupamiento (Cluster). -Para conocer grado de similitud de especies de árboles entre las parcelas evaluadas, nos basamos en la presencia-ausencia de cada especies, para ello se utilizó el coeficiente de Jaccard; $I_{j}=\mathrm{c} / \mathrm{a}+\mathrm{b}-\mathrm{c}$ (Moreno 2001). Dónde: $\mathrm{c}=$ Nro. de especies en el sitio A, $\mathrm{b}=$ Nro. de especies en el sitio B y c $=$ Nro. de especies en ambos sitios A + B. Para generar el dendrograma utilizamos el software MVPS v3.1.

\section{A nálisis d e Principales} Coordenadas.- Usamos el análisis de agrupamiento por promedio aritmético de grupos de pares no ponderados (UPGMA), para ello usamos la abundancia de especies debidamente organizadas en una matriz, esta fue corrido con el software MVPS v3.1. Para este análisis se usó índice de similaridad de Bray-Curtis: $\mathrm{BC} i \mathrm{ij}=\sum \mathrm{X}_{\mathrm{ik}}-\mathrm{X}_{\mathrm{ik} k} \sum \mathrm{X}_{\mathrm{ik}}+\mathrm{X}_{\mathrm{ik} .}$ Dónde $\mathrm{X}_{\mathrm{i} \mathrm{k}} \mathrm{y}_{\mathrm{j}_{\mathrm{k}}}=$ son las especies contadas en $\mathrm{k}$ en los muestreoiyj.

\section{Resultados}

\section{Diversidad y riqueza del bosque}

La riqueza de especies arbóreas registradas es de 277 a partir de la evaluación de cuatro hectáreas de bosque de la concesión- 
-MADERACRE S.A.C. Presenta altos valores en los indicadores de Shannon $(\mathrm{H}=4.56)$ y Alfa Fisher $\left(A_{F}=87.4\right)$. Sin embargo, a nivel de sitios de evaluación, la mayor riqueza de especies corresponde a las PPM1 y PPM3 con 146 y 145 especies respectivamente (Tabla 1 ).

Tabla 1. Indicadores de la diversidad de especímenes de flora, calculados para cada hectárea de bosque de MADERACRE SAC.

\begin{tabular}{lcccc}
\hline Parámetros & PPM 1 & PPM 2 & PPM 3 & PPM4 \\
Riqueza de especie & 146 & 110 & 145 & 118 \\
Abundancia & 498 & 568 & 555 & 392 \\
Shannon (H) & 4.29 & 3.65 & 4.28 & 4.23 \\
Equitatividad (J) & 0.86 & 0.77 & 0.85 & 0.88 \\
Alfa Fisher (AF) & 69.55 & 40.65 & 64.56 & 57.3 \\
\hline
\end{tabular}

\section{Acumulación de especies}

La acumulación de especies de flora en relación al total del área evaluada (4ha). Se observa que la curva no forma la asíntota, eso indica que existen más especies arbóreas que aún no han sido registradas en el presente estudio (Figura 2).

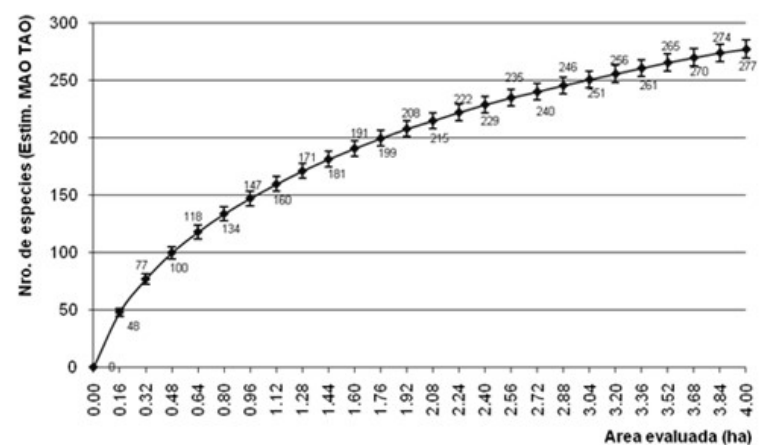

Figura 2. Curva Especie-Área para las cuatro hectáreas de bosque evaluado en la concesión MADERACRE S.A.C.

Los estimadores de riqueza, nos confirman que incrementamos el esfuerzo de muestreos en otros lugares dentro de la concesión y de similar fisonomía, se estima que se podría e $n$ C o n t r a r C $\mathrm{O} \mathrm{m} \mathrm{O}$ -mínimo 262 especies (I. Mao Tao) en 4 hectáreas de bosque y un máximo de 293 especies (al 95\% de IC de Mao Tao). En contraste, los demás estimadores son más optimistas o muestran elevados números de especies como ACE (Promedio de especies $=381$ y Desviación estándar \pm 7.8$)$, ICE (386 especies), CHAO 1 (364 \pm 19.9), CHAO $2(355 \pm 18)$, JACK $1(372 \pm 10) \mathrm{y}$ JACK 2 (411).

Del mismo modo, estos estimadores de riqueza a nivel de parcelas evaluadas muestran elevado número de especies de árboles para el bosque de MADERACRE S.A.C. (Tabla 2).

Tabla 2. Estimadores de riqueza de especies por PPM de MADERACRE SAC.

\begin{tabular}{|c|c|c|c|c|c|}
\hline Estimadore & P P M 1 & P P M 2 & P P M 3 & P P M 4 & $4 \mathrm{~h}$ a s. \\
\hline Mao Tao & 146 & 110 & 145 & 118 & 277 \\
\hline A ce & 248.7 & 164.1 & 247.8 & 189.3 & 381.44 \\
\hline I ce & 245.5 & 170.7 & 252.7 & 191.6 & 386.72 \\
\hline Chao 1 & 219.2 & 155 & 226.9 & 195 & 364.04 \\
\hline Chao 2 & 216.2 & 155.2 & 236.9 & 179.6 & 355.34 \\
\hline $\mathbf{J a c k} \mathbf{1}$ & 214.2 & 158 & 215.1 & 171.8 & 372.08 \\
\hline Jack 2 & 250.4 & 182 & 258.4 & 203 & 411.1 \\
\hline
\end{tabular}

\section{Abundancia y composición del bosque}

Especies forestales: De las 277 especies, cinco de ellas son más abundantes; Drypetes amazónica con 172 individuos ocupa el primer lugar representados por el $8.54 \%$ del total de individuos, siguen el orden decreciente Attalea phalerata (6.7\%), Quararibea wittii (5.9\%), Trichilia sp1 (4.02\%) y Pseudolmedia laevis con $3.18 \%$. Las demás especies tienen menos del $2.5 \%$ del total de la abundancia. Tabla 2 .

\section{Estructura del bosque}

La abundancia total de las cuatro PPM exhibe una "J" invertida o buena curva de equilibrio poblacional, es decir que existe alto número de individuos en las clases diamétricas menores y desciende hacia las clases diamétricas mayores - 
-(regresión exponencial negativa: $\left.\mathrm{R}^{2}=0.86\right)$. En las tres primeras clases se agrupan el $94.3 \%$ del total de individuos. La clase de 10-20 $\mathrm{cm}$ de DAP agrupan 1258 individuos que representan el $62 \%$ del total, seguido por la clase $20-30 \mathrm{~cm}(23 \%)$ y $30-40 \mathrm{~cm}$ $(8.5 \%)$. Esta distribución diamétrica de individuos indica que se cuenta con gran número de regeneración en el bosque, y en condiciones naturales está asegurada su sobrevivencia. Figura 3.

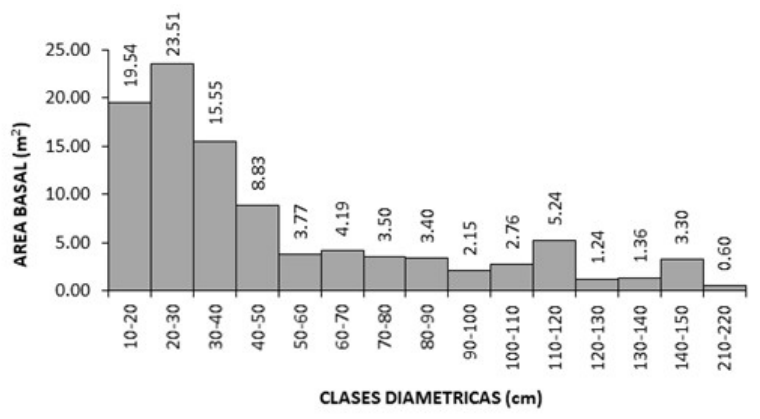

Figura 3. Distribución de la abundancia en clases diamétricas para las cuatro hectáreas de bosque de la concesión MADERACRE S.A.C.

La abundancia total de individuos agrupados en clases altimétricas tiene la forma de "campana”. Existe un alto número de tallos con alturas que oscilan de 7.5 a $15 \mathrm{~m}$ y representan el $65.2 \%$ del total de individuos. La clase 7.5-10m de altura agrupa al 30.25\% del total de individuos, seguido por la clase de $12.5-15 \mathrm{~m}(20.45 \%)$ y de $10-12.5 \mathrm{~m}$ (14.5\%) (Figura 4). El área basal total de cuatro hectáreas de bosque es $98.94 \mathrm{~m}^{2}$, que en promedio es $24.74 \mathrm{~m}^{2} /$ ha. La clase diamétrica con mayor área basal es la de $20-30 \mathrm{~cm}\left(23.5 \mathrm{~m}^{2}\right.$ de área basal y representa el $23.8 \%$ del total de Área Basal), seguido por de 10-20cm (19.7\%) y $30-40 \mathrm{~cm}$ (17.7\%) Figura 5.

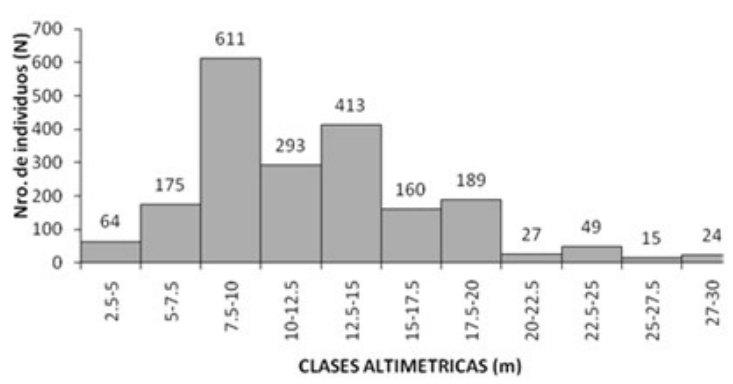

Figura 4. Distribución de la abundancia en clases altimétricas para las cuatro hectáreas de bosque de la concesión MADERACRE S.A.C.

Cabe mencionar, que en esa disminución presentan picos relevantes, como en la clase diamétrica de $110-120 \mathrm{~cm}$ que tiene $5.24 \mathrm{~m}^{2}$ (5.3\%). Las especies más dominantes son Attalea phalerata $\left(8.22 \mathrm{~m}^{2}\right.$ de área basal representa el 8.3\%), Ceiba sp1 (6.53m²; 6.6\%), Brosimum alicastrum $\left(4.66 \mathrm{~m}^{2} ; 4.7 \%\right)$, Trichilia pallida $\left(3.77 \mathrm{~m}^{2} ; 3.77 \%\right)$, Pseudolmedia laevis $\left(3.65 \mathrm{~m}^{2} ; 3.7 \%\right)$, Spondias mombin $\left(3.36 \mathrm{~m}^{2}\right.$; $3.4 \%)$, Drypetes amazonica $\left(3.27 \mathrm{~m}^{2} ; 3.3 \%\right)$, las demás tienen menos del 2.55\% delárea basal.

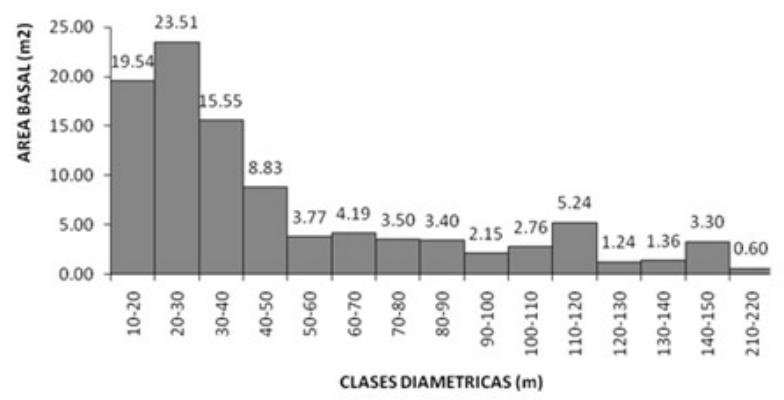

Figura 5. Distribución del área basal en clases diamétricas para las cuatro hectáreas de bosque de la concesión MADERACRE S.A.C.

Las especies forestales con mayor índice de valor de importancia (IVI) fueron; Attalea phlerata (5.73 de IVI/3), Drypetes amazonica (4.66), Trichilia pallida (3.95), Pseudolmedia laevis (2.97), Ceiba sp1 (2.96), Trichilia sp1- 
(2.68), Quararibea wittii (2.60), Brosimum alicastrum (2.20), representan el $27.75 \%$ del IVI.

Las diez familias alto Índice de Valor de Importancia de Familias (IVIF) fueron: Fabaceae (17.01 de IVI/3), Malvaceae (12.87), Meliaceae (8.65), Moraceae (8.41), Arecaceae (6.88), Putranjivaceae (5.35), Rubiaceae (3.52), Annonaceae (3.45), Sapotaceae (2.71) y Anacardiaceae (2.65) que representan el $71.50 \%$, ecológicamente, estas tienen mayor influencia sobre la dinámica del bosque.

Similaridad de especies forestales: $\mathrm{Al}$ comparar la composición de especies entre sitios de evaluación se observa desde $28 \%$ hasta $38 \%$ de similitud. Esta alta diferencia en composición de especies entre parcelas, posiblemente se deba a que las PPM están distanciadas a $2.5 \mathrm{~km}$ aproximadamente y que las especies tropicales comunes y raras de tierra firme y colinas tienen amplio rango de distribución geográfica, restringidos por los gradientes edáficos y ambientales (Figura 6).

De las 200 especies presentes en PPM1 y PPM3, solo comparten 56 especímenes $\left(I_{j}=\right.$ 0.36) y de las 166 especies distribuidas en PPM2 y PPM4, solo comparten $63\left(I_{j}=0.38\right)$ (Fig. 6). Del total de especies registradas solo 31 (11.11\% del total) están en los cuatro sitios, entre ellas destacan por su alta abundancia; Drypetes amazonica (8.54\%), Attalea phalerata (6.76\%), Trichilia pallida (5.91\%), Quararibea witti (4.17\%), Trichilia sp1 (4.02\%) y Celtis schippii $(2.29 \%)$.

La presencia de especies presenta baja distribución en las 4 PPM, esto se confirma con el Análisis de Principales Coordenadas (Figura 7).

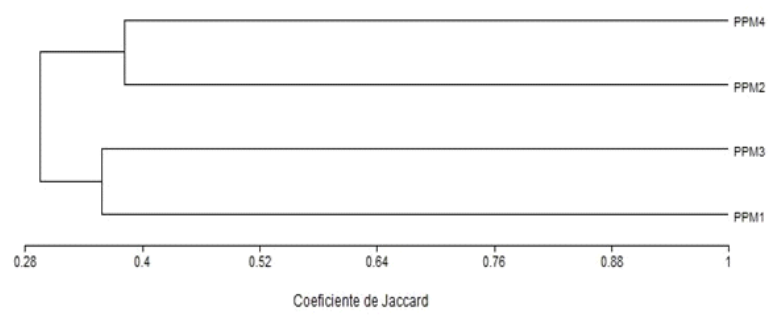

Figura 6. Dendrograma para la composición de especies de 4 PPM del bosque de MADERACRE S.A.C.

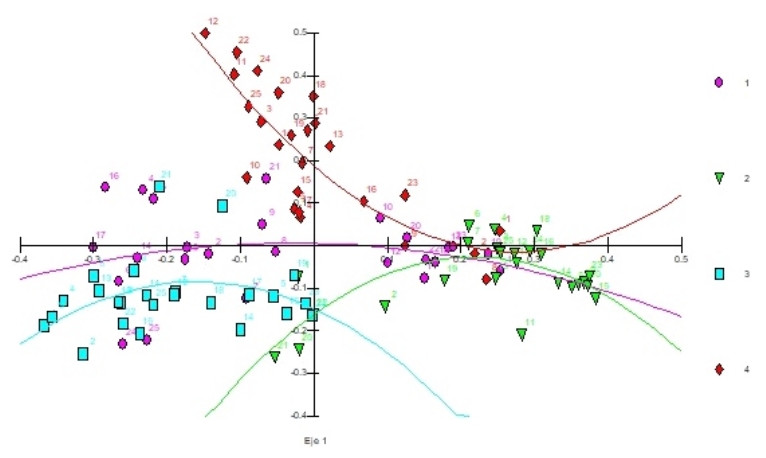

Figura 7. Distribución de las parcelas mediante el Análisis de Coordenadas Principales bajo el índice de Bray-Curtis.

\section{Discusión}

Según los índices utilizados sugieren que el bosque es diverso, el cual coincide con los bosques de la amazonia oriental (ter Steege et al. 2000, Balcazar \& Montero 2002, Balcazar 2003).

El número de parcelas evaluadas, es un estudio preliminar, que nos da una idea clara de la representatividad de especies de flora del bosque de la concesión MADERACRE S.A.C., pero es frecuente la falta de registro de la riqueza total de especies (Balcazar \& Montero 2002). Esta insuficiencia es demostrada por la inflexión de la curva de acumulación de especies-área, y otros estimadores que son más 
-forestales. A pesar de haberse evaluado 4 ha. de bosque en la citada concesión, no se logra estabilizar curva de acumulación de especies (Nebel et al.2001a, Pitman et al.2001, AraujoMurakami et al. 2005), lo que confirma que las especies de árboles de bosques de la amazonia presentan alta variación florística en una escala local (Phillips et al. 2004, ter Steege et al. 2006, Mostacedo et al. 2006), precisamente por el amplio rango de distribución espacial en la Amazonía (Pitman et al. 2001, Silman et al. 2005).

La composición florística está agrupada en diez familias Fabaceae, Malvaceae, Annonaceae, Moraceae, Rubiaceae, Lauraceae, Meliaceae, Sapotaceae y Salicaceae, la mayoría de estas fueron reportadas en otras regiones tropicales (Terborgh \& Andresen 1998, ter Steege et al. 2000; Pitman et al., 2001, Kalliola et al. 1993, Balcazar \& Montero 2002, Duque et al.2003, Mostacedo et al. 2006, ter Steege et al . 2006, Cano \& Stevenson 2009). Fabaceae también fue rica a nivel de género y especies como se reporta para otras localidades de la amazonia (Gentry 1992, ter Steege et al. 2000, 2006, Pitman et al., 2001, Kalliola et al. 1993, Balcazar \& Montero 2002, Toledo et al. 2003, Araujo-Murakami et al. 2005, Vásquez et al. 2005, Calzadilla-Tomianovich \& Cayola 2006, Cano \& Stevenson 2009) y en los bosques de Guayana (ter Steege et al. 2006). Los resultados nos sugieren que las actividades de aprovechamiento forestal, no han influido drásticamente sobre la composición florística, posiblemente la baja intensidad de cosecha volumétrica y adecuada planificación para tala de árboles maderables (Toledo et al. 2001). Estas parcelas por su reciente evaluación, no nos permiten identificar el grado de reemplazo de especies de distintos gremios, pero a través- de la similitud se confirma que las especies presentan amplio rango de distribución geográfico dentro del mismo tipo de bosque, el cual permite diferenciar los sistemas ecológicos (Josse et al. 2007) en un determinada región.

Drypetes amazónica, Attalea phalerata, Quararibea wittii y Pseudolmedia laevis fueron las más abundantes y estas especies también se distribuyen en bosques inundables (Nebel et al. 2000, Pitman et al. 2001, Araujo-Murakami et al. 2005). Sin embargo, estas especies son comunes para los bosques de colina de la Concesión MADERACRE SAC, no son las más abundantes para los bosques de tierra firme y de colina (Pitman 1999, ter Steege et al. 2000, Pitman et al. 2001, 2002, CalzadillaTomianovich \& Cayola 2006) a excepción de Attalea phalerata que registro como la segunda más importante para el bosque de pie de monte Área Natural de Manejo Integrado Madidi (Calzadilla-Tomianovich \& Cayola 2006).

El promedio de área basal de $24.74 \mathrm{~m}^{2} /$ ha registrado en el presente estudio, es inferior a los bosques tropicales (Araujo-Murakami et al. 2005, Calzadilla-Tomianovich \& Cayola 2006, Zenteno-Ruiz \& López 2010). En las clases diamétricas $10-20 \mathrm{~cm}, 20-30 \mathrm{~cm}$ son donde se agrupa el $43.5 \%$ del total de área basal y disminuye proporcionalmente en las otras clases $(\geq 15.7 \%)$, el cual nos revela la "J" invertida típico de bosques amazónicos (Pitman et al. 1999, 2001, CalzadillaTomianovich \& Cayola 2006, Díaz 2007), esta indica que apesar que realizó la tala selectiva no ha influido significativamente sobre el área basimétrica. De la misma forma, la estructura vertical del bosque está basado en la distribución altimétrica, en nuestro caso toma forma de una campana típico de bosques no- 
-perturbados, concentrándose la mayor cantidad de especies y abundancia en las clases 10-15 cm y de 15-20 m de altura (Pitman et al. 2002, Calzadilla-Tomianovich \& Cayola 2006)

Las especies Attalea phlerata, Drypetes amazonica, Trichilia pallida, Pseudolmedia laevis, Ceiba sp1, Trichilia sp1, Quararibea wittii, Brosimum alicastrum, Pseudobombax sp1, Celtis schippii, Spondias mombin, Cavanillesia umbellata, cf. Rustia 1, Drypetes sp1 y Rinorea lindeniana tienen alto peso ecológico, casi todas estas especies no están considerados para el aprovechamiento selectivo, a excepción de $B$. alicastrum. Esto indica que existe baja abundancia de las especies maderables aprovechadas, y es necesario realizar estudios para conocer su stock de regeneración natural. Asimismo, las familias Fabaceae, Malvaceae, Meliaceae, Moraceae, Arecaceae, Putranjivaceae, Rubiaceae, Annonaceae, Sapotaceae y Anacardiaceae, estas representan el $71.50 \%$ del peso ecológico total, similar a otros estudios realizados en la amazonia (Pitman et al. 2001, Kalliola et al. 1993, Balcazar \& Montero 2002).

\section{Agradecimientos}

A la empresa MADERACRE S.A.C por el financiamiento del trabajo de investigación y a los colaboradores: Nelson Kroll, Hermanos Robín y Fredy Noriega, Andrea Castagnola, Renaldo Pacheco, Rene Sebastián, Rafael Velásque, José Góngora, Marvin Montoya, José Flores y Fernando Ruiz.

\section{Literatura Citada}

Araujo-Murakami, A., Jørgensen, P. M., Maldonado, C. \& Paniagua-Zambrana, N. 2005. Composición florística y estructura del bosque de ceja de monte enYungas, sector de Tambo Quemado- Pelechuco, Bolivia. Ecología en Bolivia, Vol. 40(3): 325-338.
Balcázar, J. \& Montero, J. C. 2002. Estructura y composición florística de los bosques en el sector de Pando - Informe II. Documento Técnico 108. Proyecto de Manejo Forestal Sostenible; BOLFOR. Santa Cruz, Bolivia. 54 págs.

Balcázar, J. 2003. Estructura y composición florística de los tipos de bosques e instalación de parcelas permanentes en agrupaciones sociales del lugar (asl) del Municipio de Ixiamas - La Paz. Documento Técnico 122. Proyecto de Manejo Forestal Sostenible; BOLFOR. Santa Cruz, Bolivia. 35 págs.

BOLFOR, PROMABOSQUE. 1999. Guía para la Instalación y Evaluación de Parcelas Permanentes de Muestreo (PPMs). Santa Cruz, Bolivia.

Brako, L. \& Zarucchi, S. 1993. Catálogo de las angiospermas y gimnospermas del Perú. Monogr. Sist. Bot. Missouri Botanical Garden. 45: 1- 1286.

Britto, B. 2017. Actualización de las Ecorregiones Terrestres de Perú, propuestas en el Libro Rojo de Plantas Endémicas del Perú. Gayana Bot. 74(1): 1529

Calzadilla-Tomianovich, M. H. \& Cayola, L. 2006. Estructura y composición florística de un bosque amazónico de pie de monte, Área Natural de Manejo Integrado Madidi, La Paz - Bolivia. Ecología en Bolivia, 41(2): 117-129.

Cano, A. \& Stevenson, P. R. 2009. Diversidad y composición florística de tres tipos de bosque en la estación biológica Caparú, Vaupés. Revista Colombia ForestalVol. 12: 63-80.

Díaz, W. A. 2007. Composición florística y estructura de bosques en los asentamientos campesinos las delicias, el guamo y lechozal, estado bolívar, venezuela. Ernstia 17(1) 2007: 1-24

Duque, A., Cárdenas, D. \& Rodríguez, N. 2003. Estructural en bosques de tierra firme en el noroccidente de la Amazonía Colombiana. Caldasia 25(1): 139-152.

Encarnación, F., Zarate, R. \& Ahuite, M. 2008. Temática Vegetación en; Zonificación Ecológica y Económica del departamento de Madre de Dios. Convenio GOREMAD y IIAP. $74 \mathrm{p}$.

Gentry, A. H. 1992. Tropical forest biodiversity distributional patterns and their conservational significance. Oikos 63:19-28.

Hammer, Ø., Harper, D.A.T., Ryan, P.D. 2001. PAST: Paleontological statistics software package for education and data analysis. http://palaeoelectronica.org/2001 1/past/issue1 01.htm. 
GOREMAD \& IIAP. 2009. Macro Zonificación Ecológica Económica del departamento de Madre de Dios: Escala 1:250 000. Gobierno Regional de Madre de Dios e Instituto de Investigación de la Amazonia Peruana. $167 \mathrm{p}$.

IIAP. 2001. Propuesta de Zonificación Ecológica Económica como base para el Ordenamiento Territorial. Instituto de investigaciones de la Amazonia Peruana, Programa de Ordenamiento Ambiental. Centro regional de investigación de Madre de Dios. Puerto Maldonado - Peru. 135 p.

INADE. 2007. Mesozonificación Ecológica Económica del Corredor Interoceánico Sur, tramo Iñapari - Inambari. Instituto Nacional de Desarrollo (INADE), proyecto especial de Madre de Dios y proyecto de Estudios Automatizados Especializados - PEASE, Puerto Maldonado - Perú.

INRENA. 2003. Mapificación y evaluación forestal del bosque de producción permanente del departamento de Madre de Dios. Documento de trabajo del Instituto Nacional de Recursos Naturales del Ministerios de Agricultura. $20 \mathrm{p}$.

Josse, C., Navarro, G., Encarnación, F., Tovar, A., Comer, P., Ferreira, W., Rodríguez, F., Saito, J., Sanjurjo, J., Dyson, J., Rubin de Celis, E., Zárate, R., Chang, J., Ahuite, M., Vargas, C., Paredes, F., Castro,W., Maco, J. \& Reátegui, F. 2007. Sistemas Ecológicos de la Cuenca Amazónica de Perú y Bolivia. Clasificación y mapeo. NatureServe. Arlington, Virginia, EE UU. 96 p.

Kalliola, R., Puhakka, M. \& Dajoy, W. 1993. Amazonía peruana, vegetación húmeda en el llano subandino. Proyecto Amazonía, Universidad de Turku \& Oficina Nacional de evaluación de Recursos Naturales, Lima. 265 págs.

Lawrence, G. 1951. Taxonomy of Vascular Plants. The MacMillam. Lisboa. 823pp.

Lot, A. \& Chiang, F. 1986. Manual de herbario, administración y manejo de colecciones, técnicas de recolección y preparación de ejemplares botánicos. México. $42 \mathrm{pp}$.

Malleux, O. 1975. Mapa Forestal del Perú (Memoria explicativa). Universidad Nacional Agraria La Molina, Departamento de Manejo Forestal, Lima. 161 p. Mapa: escala $1 / 1000000$.

Manzanero, M.\& Pinelo, G. 2004. Plan silvicultural en Unidades de manejo forestal; Reserva de la Biosfera Maya, Petén, Guatemala. WWF: Programa Ambiental Regional para Centroamérica. Serie Técnica $\mathrm{N}^{\circ} 3.49 \mathrm{p}$.
Matteucci, S. \& Colma,A.1982. Metodología para el estudio de la vegetación. Ed. Eva. Chesneau, Washinton DC. Págs.: 3-150.

Moreno, C. E. 2001. Métodos para medir la biodiversidad. M\&T-Manuales y Tesis SEA, vol. 1. Zaragoza, $84 \mathrm{p}$.

Mostacedo, B., Balcázar, J. \& Montero, J. C. 2006. Tipos de bosque, diversidad y composición florística en la Amazonia sudoeste de Bolivia. Ecología en Bolivia, 41(2): 99-116

Nebel, G., Kvist, L. P, Vanclay, J. K., Christensen, H., Freitas, L. \& Ruiz J. 2000. Estructura y composición florística del bosque de la llanura aluvial en la Amazonía peruana: I. El bosque alto. IIAP. Folia AmazónicaVol. 10 (1-2)

Nebel, G., Kvista, l. P., Vanclay, J.K., Christensen, H., Freitasd, L., \& Ruize, J. 2001(a). Structure and floristic composition of flood plain forests in the Peruvian Amazon I. Overstorey. Forest Ecology and Management 150:27-57

Phillips, O., Baker, T., Arroyo, L., Higuchi, N., Killeen, T., Laurance, W. F., Lewis, S. L., Lloyd, J., Malhi, Y., Monteagudo, A., Neill, D., Nuñez, P., Silva, N., Terborgh, T., Vásquez, R., Alexiades, M., Almeida, S., Brown, S., Chave, J., Comiskey, J.A., Czimczik, C. I., Di Fiore, A., Erwin, T., Kuebler, C., Laurance, S. G., Nascimento, E. M., Olivier, J., Palacios, W., Patiño, S., Pitman, N., Quesada, C. A., Saldias, M., Torres, A. \& Vinceti, B. 2004. Pattern and process in Amazon tree turnover, 1976-2001. The Royal Society. 27 p.

Pitman, N. C. A., Terborgh, J., Silman, M. R. \& Núñez, P. 1999. Tree species distributions in an upper Amazonian forest. Ecology. 80(8): 2651-61

Pitman, N. C. A., Terborgh, J. W., Núñez, P. \& Silman, M. R. 2001. Especies comunes de la parte baja de Madre de Dios, Perú. Pp. 46-52. En: L. Rodríguez. El Manu y Otras experiencias de Investigación y Manejo de Bosques Neotropicales. Proyecto Aprovechamiento y Manejo Sostenible de la Biosfera del Manu, Cuzco. 301 p.

Pitman, N. C. A., Terborgh, J. W., Silman, M. R., Nuñez, P., Neill, D. A., Ceron, C. E., Palacios,W. A. \& Aulestia, M. 2002. A comparison of tree species diversity in two upper Amazonian forests. Ecology, 83(11), 2002,pp. 3210-3224. 
Poorter, L., Boot, R. G. A., Hayashida-Oliver, Y., Leigue-Gomez, J., Peña-Claros, M. \& Zuidema, P. A. 2001. Dinámica de Especies Arbóreas en un Bosque Húmedo Tropical en el Norte de la Amazonía Boliviana. En Mostacedo B. y Fredericksen T. Regenracion y Silvicultura de bosques tripicales de Bolivia. Proyecto de Manejo Forestal Sostenible (BOLFOR). Santa Cruz, Bolivia. 53-76

Ribeiro, J., Hopkins, M., Vicentini, A., Sothers, C., Costa, M., Brito, J., Souza, M., Martins, I., Iohmann, I., Assunção, P., Pereira, E., Silva, C., Mesquita, M. \& Procópio, L. 1999. Flora da Reserva Ducke: guia de identificação das plantas vasculares de uma floresta de terra firme na Amazônia Central. MANAUS. 800 p.

Silman, M. R., Araujo-Murakami, A., Urrego, D. H., Bush, M. B. \& Pariamo, H. 2005. Estructura de las comunidades de árboles en el límite sur de la Amazonía occidental: Manu y Madidi. Ecología en Bolivia,Vol.40(3): 443-452

ter Steege, H., Pitman, N. C. A., Phillips, O. L., Chave, J., Sabatier, D., Duque, A., JeanFrancois, M., Prévost, M. F., Spichiger, R., Castellanos, H., von Hildebrand, P. \& Vásquez, R. 2006. Continental-scale patterns of canopy tree composition and function across Amazonia. Nature, Vol. 443:28.

ter Steege, H., Sabatier, D., Castellanos, H., van Andel, T., duivenvoorden, J., de Oliveira, A. A., Ek, R., Lilwah, R., Maas, P. \& Mori, S. 2000. An analysis of the floristic composition and diversity of Amazonian forests including those of the Guiana Shield. J.Trop. Ecol. 16, 801--828.

Toledo, M., Fredericksen, T., Licona, J. C. \& Mostacedo, B. 2001 . Impactos del aprovechamiento forestal en la flora de un bosque semideciduo pluviestacional de Bolivia. Documento Técnico 106. Proyecto de Manejo Forestal Sostenible; BOLFOR. Santa Cruz, Bolivia. 23 p.

Vallejo-Joyas, M. I., Londoño-Vega, A. C., LópezCamacho, R., Galeano, G., Álvarez-Dávila, E. \& Devia-Álvarez, W. 2005. Establecimiento de parcelas permanentes en bosques de Colombia. Instituto de Investigación de Recursos Biológicos Alexander von Humboldt. Bogotá D. C., Colombia. 310 p. (Serie: Métodos para estudios ecológicos a largo plazo; No. 1).
Vásquez, R., Rojas, R., Monteagudo, A., Meza, K., Van Der Werff, H., Ortiz-Gentry R. \& Catchpole, D. 2005. Flora Vascular de la selva central del Perú: Una aproximación de la composición florística de tres Áreas Naturales Protegidas. ARNALDOA 12 (1-2): 112-125.

WWF, 2004. Monitoreo ecológico del manejo forestal en el trópico húmedo: Una guía para operadores forestales y certificadores con énfasis en Bosques de Alto Valor para la Conservación (BAVC). Fondo Mundial para la Naturaleza-Centroamérica. 124 págs.

Zenteno-Ruiz, F. S. \& López, R. P. 2010. Composición, estructura y patrón espacial de un bosque Tucumano Boliviano en el departamento de Tarija (Bolivia). Darwiniana 48(1): 32-44. 
